\title{
The influence of dietary carbohydrate and pre- exercise glucose consumption on supramaximal intermittent exercise performance
}

\author{
D. G. Jenkins PhD, C. A. Hutchins BHMS(Ed) (Hons) and D. Spillman BHMS(Ed) Grad Dip \\ Nutr Diet \\ Human Performance Laboratory, Department of Human Movement Studies, The University of Queensland, \\ Australia
}

\begin{abstract}
The present study examined whether a pre-exercise consumption of glucose by subjects having adhered to a 3-day low carbohydrate (CHO) or normal CHO diet would influence supramaximal intermittent exercise performance. Sixteen moderately active men volunteers (mean(s.d.) age 20.0(1.3) years) agreed to undertake three exercise tests over an 8-day period; in addition to completing a $\dot{V}_{\mathbf{O}_{2} \max }$ test, the subjects performed two identical maximal interval tests $\left(\mathrm{MIT}_{1}\right.$ and $\left.\mathrm{MIT}_{2}\right)$. Periods of 3 days separated each of the three tests. The interval tests involved five 60 -s 'all-out' cycling bouts working against a resistance of $0.075 \mathrm{~kg} \mathrm{~kg}^{-1}$ body mass; each bout was separated by 5 min of passive recovery. For 3 days preceding the first interval test $\left(\mathrm{MIT}_{1}\right)$, all subjects adhered to a 'moderate' $\mathrm{CHO}$ diet which comprised $59.1 \%$ (approximately $4.1 \mathrm{~g} \mathrm{~kg}^{-1}$ body mass) of the daily energy intake as CHO. Following MIT $_{1}$ and for 3 days before $\mathrm{MIT}_{2}$ subjects were randomly assigned to follow either a moderate CHO diet $(\mathbf{6 0 . 8 \% )}$ or a low CHO diet $\mathbf{( 1 4 . 4 \%}$ or $1.1 \mathrm{~g} \mathrm{~kg}^{-1}$ body mass). All food and drink consumed during the experimental period was weighed and recorded for later dietary analysis. One hour before $\mathrm{MIT}_{2}$, eight subjects were administered (in single blind fashion) a $15 \%$ glucose solution ( $1 \mathrm{~g} \mathrm{~kg}^{-1}$ body mass) while the other eight subjects consumed a low-energy sweetened placebo. During both interval tests, values of work, exercise $\dot{V}_{\mathrm{O}_{2}}$, plasma glucose, plasma lactate and venous blood $\mathrm{pH}$ were statistically analysed. No changes in performance between $\mathrm{MIT}_{1}$ and $\mathrm{MIT}_{2}$ across conditions were found $(P>0.05)$. However, those subjects who consumed the glucose solution before $\mathrm{MIT}_{2}$ (irrespective of their dietary CHO intake) consumed significantly less oxygen during exercise than those who had been given the placebo solution $(P<0.05)$. While these findings question the ergogenic potential of consuming glucose before supramaximal exercise, the $\dot{V}_{\mathrm{O}_{2}}$ data implicate a possible shift in substrate utilization during repeated sprint exercise after pre-exercise glucose ingestion.
\end{abstract}

Keywords: Supramaximal exercise, dietary carbohydrate, glucose ingestion
Researchers have long recognized the intimate relationship between exercise intensity and carbohydrate (CHO) utilization. Yet, when compared with the volume of literature related to $\mathrm{CHO}$ availability and submaximal exercise performance, relatively little is known of the influence which dietary CHO has on supramaximal exercise capacity. At intensities above the anaerobic threshold, electrolyte displacement, intramuscular acidosis and possible failure of the calcium pumps to return calcium to the sarcoplasmic reticulum have been implicated as the principal factors limiting high intensity exercise performance ${ }^{1}$. None the less, a number of recent studies have shown that exercise time to exhaustion at intensities approximating $100 \% \dot{V}_{\mathrm{O}_{2} \max }$ can be extended using dietary strategies intended to enhance pre-exercise intramuscular glycogen stores ${ }^{2,3}$. Maughan and Poole $^{2}$ showed that when compared with performance following a low $\mathrm{CHO}$ diet $(2.6 \% \mathrm{CHO})$, time to fatigue at $104 \% \dot{V}_{\mathrm{O}_{2} \max }$ could be doubled with a high $\mathrm{CHO}(84 \%)$ diet (from $3.32 \mathrm{~min}$ to $6.65 \mathrm{~min}$ ). A recent investigation conducted in our laboratory also found that when compared with a low CHO diet $(12 \%$ $\mathrm{CHO})$, a moderate 3 -day intake of dietary $\mathrm{CHO}(58 \%$ $\mathrm{CHO})$ significantly improved total work accomplished over five 60-s 'all-out' cycling bouts ${ }^{4}$. Again with reference to submaximal exercise, a number of researchers have found that when glycogen reserves are critically low during the latter stages of prolonged submaximal exercise, ingestion of glucose can delay the onset of fatigue and help support continued exercise at a relatively high power output ${ }^{5,6}$. Having recently confirmed that dietary $\mathrm{CHO}$ can significantly influence supramaximal interval performance, the present investigation was undertaken to establish whether pre-exercise consumption of glucose would influence supramaximal exercise performance of subjects who had followed a strategy intended either to maintain or reduce $\mathrm{CHO}$ availability at the muscle.

\section{Subjects and methods}

Address for correspondence: Dr D. G. Jenkins, Human Performance Laboratory, Department of Human Movement Studies, The University of Queensland, 4072, Australia (C) 1994 Butterworth-Heinemann Ltd 0306-3674/94/030171-06 


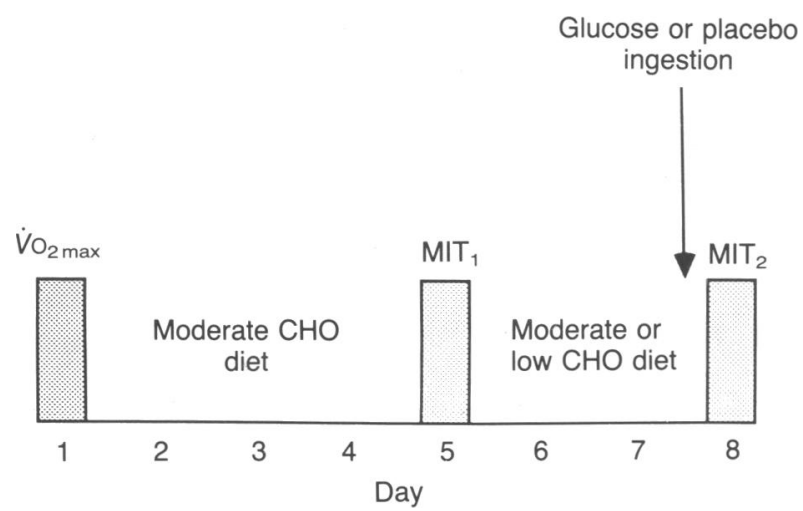

Figure 1. Overview of the 8-day experimental period: $\mathrm{CHO}$, carbohydrate; $\mathrm{MIT}_{1}, \mathrm{MIT}_{2}$, maximal interval tests

three tests, and all food and drink consumed during this period was weighed and recorded for later dietary analysis. Between the $\dot{V}_{\mathrm{O}_{2} \text { max }}$ test and the first interval test $\left(\mathrm{MIT}_{1}\right)$, all subjects adhered to a moderate $\mathrm{CHO}$ intake (59\% $\mathrm{CHO}$ ). However, on completion of $\mathrm{MIT}_{1}$ and for the 3 days preceding $\mathrm{MIT}_{2}$ subjects were matched according to their $\mathrm{MIT}_{1}$ performance and assigned to follow either a moderate $\mathrm{CHO}$ diet $(60 \% \mathrm{CHO})$ or a low $\mathrm{CHO}$ diet $(12 \%$ $\mathrm{CHO}$ ). One hour before $\mathrm{MIT}_{2}$ four subjects in both dietary groups were given a $15 \%$ glucose solution $\left(1 \mathrm{~g} \mathrm{~kg}^{-1}\right.$ body mass) while four others from both dietary groups consumed a low-energy sweetened placebo. Measures of work, exercise $\mathrm{V}_{2}$, venous blood $\mathrm{pH}$, plasma lactate and plasma glucose from $\mathrm{MIT}_{1}$ and $\mathrm{MIT}_{2}$ were compared across dietary groups and between the pre-exercise solutions consumed, making it possible to evaluate the influence of pre-exercise glucose consumption and dietary $\mathrm{CHO}$ upon supramaximal exercise performance. With the exception of the three exercise tests imposed during the experimental period, subjects refrained from all other exercise. Exercise testing was conducted on a basket-loading mechanically braked cycle ergometer (Monark 814E; Monark, Varberg, Sweden).

Sixteen recreationally active men students volunteered to take part in the study which had approval from The University of Queensland Human Subjects Ethical Review Committee. After undergoing a medical examination and providing informed consent, the subjects (mean(s.d.) age 20.1(1.3) years) undertook two familiarization sessions. One session included incremental exercise to volitional fatigue, identical to the procedure to be employed in the maximal oxygen uptake $\left(\dot{V}_{\mathrm{O}_{2}} \max \right)$ test, while the other required the subjects to cycle five 60-s all-out sprints, replicating the interval test which is described below.

\section{Determination of maximal oxygen uptake}

Using a procedure modified from that described by Taylor et al. ${ }^{7}, \dot{V}_{\mathrm{O}_{2} \max }$ was established 3 days before the first maximal interval test $\left(\mathrm{MIT}_{1}\right)$. Immediately before testing, each subject completed a 5-min warm-up, cycling at $90 \mathrm{~W}(60 \mathrm{r} . p . \mathrm{m}$. at $15 \mathrm{~N})$. This was followed by $3 \mathrm{~min}$ of stretching. Using the response to exercise during the practice sessions, the initial workload for each individual was chosen to approximate $70-80 \% \dot{V}_{\mathrm{O}_{2} \max }$. Subjects maintained each workload for $3 \mathrm{~min}$ and, after each 3-min period (during which the pedalling rate was maintained at 60 r.p.m.) additional resistance $(5 \mathrm{~N})$ was applied.

The first 60 -s collection period began at $2 \mathrm{~min}$ (i.e. during the final minute of each workload), with further data sampled at 3-min intervals until the subject, nearing exhaustion, gave a clear signal that he was capable of only a further $60 \mathrm{~s}$ of exercise. The final data were collected during this period.

A plateau reflecting less than a $100 \mathrm{ml}$ increase in $\dot{V} \mathrm{O}_{2}$ with a $30 \mathrm{~W}$ increase in workload was accepted as evidence that maximal oxygen uptake had been achieved. Samples of expired air were collected in 200-1 Douglas bags downstream from a mixing chamber adjacent to the mouthpiece (Hans Rudolph, Kansas City, USA; Model 2700); expired air was analysed following the test. Calibration of the gas analysers (Amatek, Pittsburgh, USA; SOV S-3A/1, and $\mathrm{COV}$ CD3A) was carried out immediately before and after each test using a certified gravimetric gas mixture (Commonwealth Industrial Gases, Brisbane, Australia). Minute volume was measured using a chain compensated Tissot tank (Warren E. Collins, Massachusetts, USA) and values of $\dot{V}_{\mathrm{O}_{2}}$ were expressed as $1 \mathrm{~min}^{-1}$ and $\mathrm{ml} \mathrm{kg}^{-1} \mathrm{~min}^{-1}$, corrected to standard temperature, pressure dry.

\section{Maximal interval tests $\left(\mathrm{MIT}_{\mathbf{1}}\right.$ and $\mathrm{MIT}_{\mathbf{2}}$ )}

Three days after the $\dot{V}_{\mathrm{O}_{2} \text { max }}$ test, the subjects arrived at the laboratory in a post-absorptive condition to be weighed and to undertake the first interval test. Subjects had been asked not to eat within $4 \mathrm{~h}$ of exercise. The test entailed five 60-s all-out cycling bouts with each bout separated by $5 \mathrm{~min}$ of passive seated recovery. Subjects were instructed to register the maximum possible number of pedal revolutions in each bout while cycling against a resistance of $0.75 \mathrm{~kg} \mathrm{~kg}^{-1}$ body mass.

Before the warm-up and during the last $30 \mathrm{~s}$ of each recovery period, duplicate samples of finger-tip capillary blood $(20 \mu \mathrm{l})$ were collected and centrifuged. The capillary tubes were then cut above the plasma/haematocrit interface before the plasma was resealed and stored at $4^{\circ} \mathrm{C}$ for the later analysis of lactate and glucose. The highest lactate and glucose values registered during the 25-min test were accepted as the peak concentrations and were entered for data analysis. Peak values were expected to occur at different times between individuals over the 25-min period. In addition, before the warm-up and within $3 \mathrm{~min}$ following the fifth and final bout, $5 \mathrm{ml}$ of venous blood was sampled from an anticubital vein. Blood $\mathrm{pH}$ was immediately measured using an ionized calcium analyser (ABL2; Radiometer, Copenhagen, Denmark) which had been calibrated using known standards within the range of mean(s.d.) expected readings $(7.383(0.005)$ and $6.841(0.005)$ at $\left.37^{\circ} \mathrm{C}\right)$.

Insertion of the mouthpiece and attachment of the noseclip preceded exercise by $10 \mathrm{~s}$, allowing for expired air to be analysed during the five exercise bouts. Ten seconds had been established as sufficient to clear the dead space from the system before first 
collection. Minute volume of inspired air was measured by a turbine ventilometer (Morgan, UK), while expired air was sampled in Douglas bags for later analysis. Measures of bout-by-bout and total oxygen consumption during each interval test were included for data analysis.

\section{Dietary control}

Between the $\dot{V}_{\mathrm{O}_{2 \max }}$ test and $\mathrm{MIT}_{2}$ the subjects' diets were closely monitored. For the first 3-day period, diets provided $59 \%$ of the daily energy intake in the form of the CHO. This approximated $4.1 \mathrm{~g} \mathrm{~kg}^{-1}$ body mass and was intended to 'normalize' muscle glycogen stores for all subjects before $\mathrm{MIT}_{1}$. Following $\mathrm{MIT}_{1}$ diets provided either $60 \%$ of the daily energy intake in the form of $\mathrm{CHO}$ (approximately $4.1 \mathrm{~g} \mathrm{~kg}^{-1}$ body mass) or $14 \%$ (approximately $1.1 \mathrm{~g} \mathrm{~kg}^{-1}$ body mass) of the energy intake as CHO. The low $\mathrm{CHO}$ intake for eight of the 16 subjects was intended to reduce muscle glycogen resynthesis following $\mathrm{MIT}_{1}$, but at the same time to provide sufficient $\mathrm{CHO}$ to minimize the risk of changes in resting circulatory acid-base balance (as described by Greenhaff et al. ${ }^{9}$ ). The total caloric intake of each subject's diet was calculated according to his body weight and expected activity level using the Schofield equation.

Known quantities and nutritional content of all food and drink taken by the 16 subjects during the experimental period were provided by the researchers. None the less, subjects were issued with digital weighing scales (Arlec, Taiwan) and requested to weigh and accurately record all food and drink they consumed. Dietary analysis was achieved using the Diet 2 software package (Xyrus Software, Brisbane, Australia) which yielded a comprehensive history of all nutrients taken.

\section{Analysis of lactate and glucose}

Concentrations of plasma lactate were determined using reagent solutions supplied by Boehringer Mannheim, Lewes, UK. Fluorescence was determined spectrophotometrically at $340 \mathrm{~nm}$ with a 11-Multistat, Micro Centrifugal Analyser (discrete system analyser, Model MS2). Known standards supplied by the manufacturer made both calibration and quality control of the instrument possible before analysis. Plasma glucose was assayed from $10 \mu \mathrm{l}$ of plasma using a Kodak Ektachem DT slide (Eastman Kodak, Rochester, New York, USA).

\section{Statistical analysis}

Total work accomplished, oxygen consumed during the interval test, peak plasma lactate, peak glucose concentrations and venous blood $\mathrm{pH}$ were analysed as the study's dependent variables. Before $\mathrm{MIT}_{1}$ dietary treatment for all subjects $(n=16)$ was identical; subjects adhered to a moderate $\mathrm{CHO}$ diet for 3 days and did not consume a glucose or placebo drink before undertaking $\mathrm{MIT}_{1}$. The dependent variables from $\mathrm{MIT}_{1}$ were therefore used as controls, against which changes in the same measures during
$\mathrm{MIT}_{2}$ were statistically compared. Analysis of variance assessed changes across four conditions: (1) moderate $\mathrm{CHO}$ diet and pre-exercise ingestion of glucose (Mod/glu; $n=4)$; (2) moderate CHO diet and pre-exercise ingestion of a low-energy sweetened placebo (Mod/pla; $n=4)$; (3) low dietary intake of $\mathrm{CHO}$ and pre-exercise glucose ingestion (Low/glu: $n=4)$; and (4) low CHO diet and pre-exercise consumption of a sweetened low-energy placebo (Low/pla; $n=4)$. Newman Keuls post-hoc analysis was used to locate any differences identified by the analysis of variance, and significance was accepted at $P=0.05$.

\section{Results}

As shown in Table 1, diets for the 16 subjects between the $\dot{V} \mathrm{O}_{2 \max }$ test and $\mathrm{MIT}_{1}$ comprised a mean(s.d.) $59.1(3.8) \%$ of the total energy intake as $\mathrm{CHO}$, a figure which approximated $4.1(0.7) \mathrm{g} \mathrm{kg}^{-1}$ body mass. Protein and fat accounted for $24 \%$ and $17 \%$ respectively of the remaining calories consumed. Between $\mathrm{MIT}_{1}$ and $\mathrm{MIT}_{2}$ those subjects remaining on the normal CHO diet averaged $60.8 \%, 23.1 \%$ and $16.1 \%$ of their calories in the form of $\mathrm{CHO}$, protein and fat respectively, with a mean(s.d.) $\mathrm{CHO}$ intake of $4.4(0.8) \mathrm{g} \mathrm{kg}^{-1}$ body mass a day. Those who followed the low CHO diet consumed $14.4 \%, 27.3 \%$ and $59.3 \%$ of their calories as $\mathrm{CHO}$, protein and fat respectively; the daily $\mathrm{CHO}$ intake for these eight subjects corresponded to a mean(s.d.) $1.1(0.2) \mathrm{g} \mathrm{kg}^{-1}$ body mass. The recommended daily intake of all vitamins and minerals during both periods of dietary control were met by all diets. Table 2 provides a summary of the subjects' physical characteristics together with their measures of maximal oxygen uptake.

Despite significant differences in $\mathrm{CHO}$ intake for eight of the subjects between pre-MIT ${ }_{1}$ and pre-MIT 2 $(P<0.01)$, total work accomplished between $\mathrm{MIT}_{1}$ and $\mathrm{MIT}_{2}$ showed no significant change in response to either dietary $\mathrm{CHO}$ or glucose solution (Figure 2). Bout by bout analysis of work also failed to identify differences across the conditions.

The demanding nature of the interval test is reflected by the fatigue profile, especially from bout 1 to bout 3 (Figure 3). Work over these three intervals showed a mean decrease of $33 \% \quad(P<0.01)$ but thereafter appeared to plateau with an average decrease in performance of only $5.5 \%(P>0.05)$ being recorded between bouts 3 and $5(n=16)$.

Table 1. Subjects' carbohydrate $(\mathrm{CHO})$ intake pre-MIT ${ }_{1}$ and pre-MIT

\begin{tabular}{cc}
\hline \multicolumn{2}{c}{ CHO Intake (\%) } \\
\cline { 2 - 3 } Pre-MIT, & Pre-MIT \\
\hline $59.1(3.8)(n=16)$ & $\begin{array}{l}60.8(1.9)(n=8) \\
14.4(3.9)(n=8)\end{array}$ \\
\hline
\end{tabular}

Values are mean(s.d.) 
Table 2. Mean(s.d.) weight, age and values of maximal oxygen uptake for the 16 subjects

\begin{tabular}{|c|c|c|c|c|}
\hline \multirow[b]{2}{*}{ Subject } & \multirow[b]{2}{*}{ Weight (kg) } & \multirow[b]{2}{*}{ Age (years) } & \multicolumn{2}{|c|}{$\dot{V}_{O_{2 \max }}$} \\
\hline & & & $\left(I \min ^{-1}\right)$ & $\left(\mathrm{m} / \mathrm{kg}^{-1} \mathrm{~min}^{-}\right.$ \\
\hline 1 & 73.1 & 19 & 4.24 & 58.08 \\
\hline 2 & 78.4 & 21 & 4.43 & 56.79 \\
\hline 3 & 59.5 & 20 & 2.86 & 48.40 \\
\hline 4 & 64.0 & 18 & 4.02 & 63.31 \\
\hline 5 & 71.5 & 22 & 3.58 & 44.25 \\
\hline 6 & 80.8 & 19 & 4.53 & 56.70 \\
\hline 7 & 82.1 & 22 & 3.58 & 44.25 \\
\hline 8 & 76.2 & 21 & 3.81 & 50.91 \\
\hline 9 & 82.8 & 18 & 4.43 & 54.42 \\
\hline 10 & 66.4 & 18 & 4.00 & 58.08 \\
\hline 11 & 72.5 & 20 & 3.67 & 50.90 \\
\hline 12 & 69.5 & 20 & 3.15 & 45.50 \\
\hline 13 & 89.0 & 22 & 4.01 & 45.00 \\
\hline 14 & 67.4 & 21 & 4.09 & 61.15 \\
\hline 15 & 77.5 & 20 & 5.17 & 67.08 \\
\hline 16 & 75.5 & 20 & 4.26 & 56.86 \\
\hline Mean(s.d.) & $74.1(7.4)$ & $20(1.3)$ & $3.94(1.7)$ & $53.50(7.5)$ \\
\hline
\end{tabular}

Unfortunately, changes in power output within each 60 -s bout could not be measured.

Although dietary $\mathrm{CHO}$ had no significant influence on exercise oxygen consumption between $\mathrm{MIT}_{1}$ and $\mathrm{MIT}_{2}$ when glucose and placebo solutions were compared, irrespective of $\mathrm{CHO}$ intake, a significant difference in oxygen consumption emerged $(P<0.05)$. Figure 4 shows that those subjects who ingested the placebo drink $1 \mathrm{~h}$ before $\mathrm{MIT}_{2}$ consumed more total oxygen during exercise than those who ingested the $15 \%$ glucose solution pre-MIT 2 . However, post-hoc analysis failed to identify differences in exercise $\dot{V}_{\mathrm{O}_{2}}$ between individual bouts.

From blood sampled $5 \mathrm{~min}$ before exercise, it was found that glucose ingestion $1 \mathrm{~h}$ pre--MIT 2 significantly raised the mean(s.d.) resting plasma glucose concentrations from $4.1(0.1) \mathrm{mmol}^{-1}$ to $5.0(0.8) \mathrm{mmol}^{-1}$ $(P<0.05 ; n=8)$. However, from blood sampled

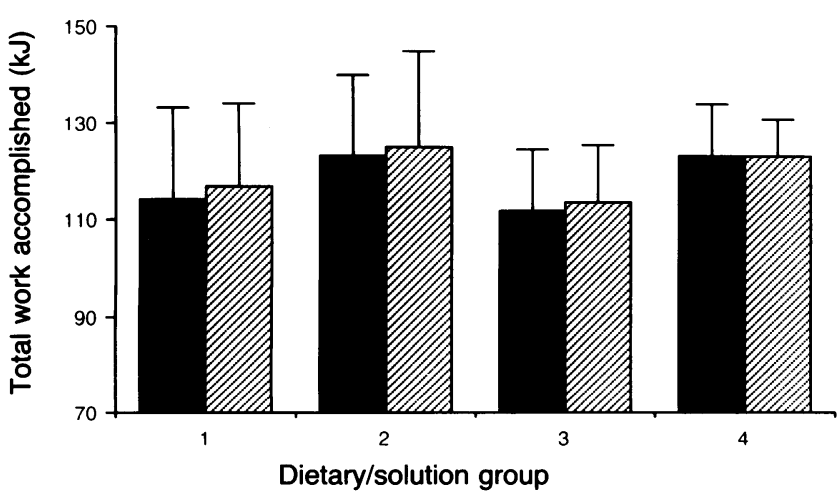

Figure 2. Mean(s.d.) totals of work accomplished ( $\square$, $\mathrm{MIT}_{1}$; and $囚, \mathrm{MIT}_{2}$ ) by each of the four groups being compared: Mod/glu, moderate carbohydrate diet and pre-MIT ${ }_{2}$ glucose consumption; Mod/pla, moderate carbohydrate diet and pre-MIT 2 placebo consumption; Low/glu, low carbohydrate diet and pre-MIT 2 , glucose ingestion; Low/pla, low carbohydrate diet and pre-MIT placebo consumption. No significant differences in work output between the four groups were found

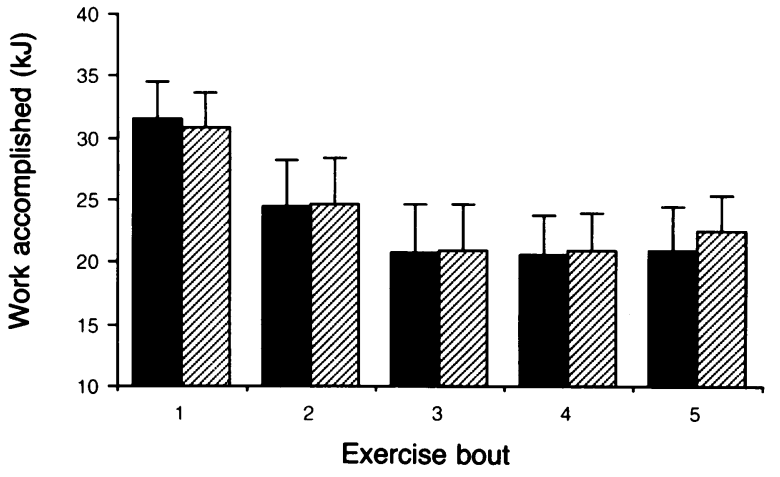

Figure 3. Mean(s.d.) work accomplished in each of the five exercise bouts (maximal interval tests, $\mathrm{MIT}_{1}$ and $\mathrm{MIT}_{2}$ ) by the 16 subjects. Fatigue from bouts one to three averaged $33 \%$, while work output fell by a mean of $5 \%$ over the final three bouts

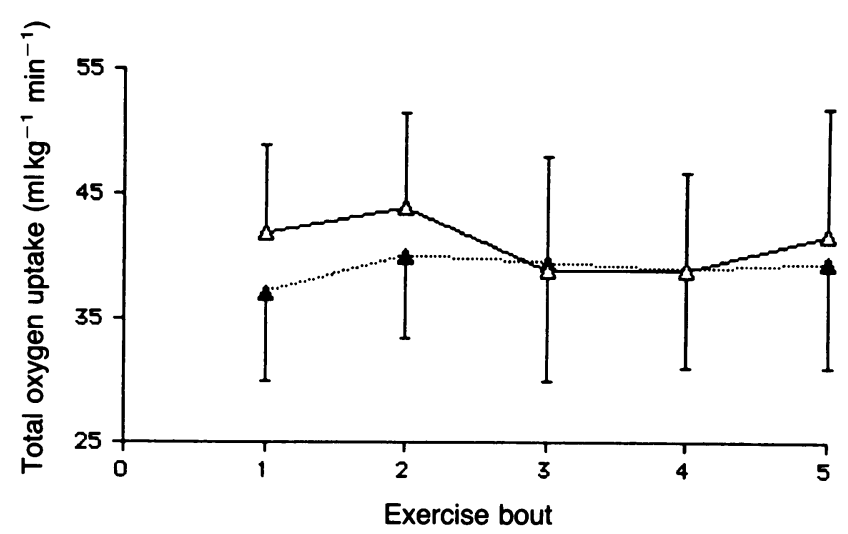

Figure 4. Bout-by-bout mean (s.d.) oxygen consumption for subjects who consumed the $15 \%$ glucose solution and those who consumed the low-joule placebo before undertaking $\mathrm{MIT}_{2}: \Delta$, placebo $(n=8) ; \Delta$, glucose $(n=8)$

during each recovery bout, differences across conditions with respect to exercise-induced changes in peak plasma glucose concentrations were not found. Similarly, dietary $\mathrm{CHO}$ and pre-MIT 2 solution exerted no significant influence either on resting or exercise plasma lactate concentrations. Table 3 shows the subjects' $\mathrm{MIT}_{1}$ and $\mathrm{MIT}_{2}$ resting and peak concentrations of plasma glucose and plasma lactate across the four conditions. Even though blood $\mathrm{pH}$ fell from a resting mean(s.d.) value of 7.32(0.02) to $7.05(0.06)$ in response to the interval tests $(n=16)$, resting and post-exercise measures of blood $\mathrm{pH}$ showed no change in response to diet or solution. The low $\mathrm{CHO}$ diet did not induce a significant change in pre-exercise blood $\mathrm{pH}$ before $\mathrm{MIT}_{2}$ suggesting that resting circulatory acid-base balance had been preserved.

\section{Discussion}

The aim of the present investigation was to evaluate the influence of pre-exercise glucose consumption on supramaximal intermittent exercise performance with subjects having adhered to either a low carbohydrate (CHO) or moderate $\mathrm{CHO}$ diet for 3 days. The principal finding is that neither dietary $\mathrm{CHO}$ intake nor 
Glucose ingestion and supramaximal exercise: D. G. Jenkins et al.

Table 3. Resting and peak concentrations of plasma glucose and plasma lactate (MIT 1 and $\left.\mathrm{MIT}_{2}\right)$

\begin{tabular}{|c|c|c|c|c|c|c|c|c|}
\hline \multirow[b]{3}{*}{ Group } & \multicolumn{4}{|c|}{ Plasma glucose concentration ( $\left.\mathrm{mmoll}^{-1}\right)$} & \multicolumn{4}{|c|}{ Plasma lactate concentration $\left(\mathrm{mmol}^{-1}\right)$} \\
\hline & \multicolumn{2}{|c|}{$M I T_{1}$} & \multicolumn{2}{|c|}{$M I T_{2}$} & \multicolumn{2}{|c|}{$M I T_{1}$} & \multicolumn{2}{|c|}{$M I T_{2}$} \\
\hline & Rest & Peak & Rest & Peak & Rest & Peak & Rest & Peak \\
\hline \multicolumn{9}{|l|}{ Mod/g/u } \\
\hline 1 & 4.2 & 8.7 & 4.3 & 8.1 & 2.3 & 19.5 & 3.4 & 19.7 \\
\hline 2 & 4.3 & 6.9 & 4.4 & 6.2 & 1.7 & 24.3 & 3.1 & 17.1 \\
\hline 3 & 4.3 & 7.1 & 5.4 & 5.9 & 2.4 & 20.8 & 4.0 & 20.7 \\
\hline 4 & 4.4 & 9.2 & 4.5 & 8.2 & 3.1 & 18.7 & 2.9 & 18.6 \\
\hline Mean(s.d.) & $4.3(0.1)$ & $7.9(0.9)$ & $4.7(0.4)$ & $7.1(1.1)$ & $2.4(0.5)$ & $20.8(2.3)$ & $3.4(0.4)$ & $19.0(1.3)$ \\
\hline \multicolumn{9}{|l|}{ Mod/pla } \\
\hline 5 & 3.9 & 6.5 & 3.7 & 5.7 & 3.2 & 21.0 & 3.0 & 22.1 \\
\hline 6 & 4.0 & 6.6 & 3.2 & 7.5 & 3.2 & 20.2 & 3.0 & 18.1 \\
\hline 7 & 3.2 & 4.1 & 3.4 & 4.8 & 2.5 & 16.8 & 1.5 & 16.3 \\
\hline 8 & 5.4 & 9.9 & 5.3 & 8.3 & 1.1 & 22.1 & 1.5 & 23.1 \\
\hline Mean(s.d.) & $4.1(0.8)$ & $6.8(2.1)$ & $3.9(0.8)$ & $6.6(1.4)$ & $2.5(0.8)$ & 20.2(1.9) & $2.3(0.8)$ & $19.9(2.8)$ \\
\hline \multicolumn{9}{|l|}{ Low/glu } \\
\hline 9 & 3.7 & 6.3 & 7.0 & 6.6 & 1.8 & 18.4 & 6.3 & 23.7 \\
\hline 10 & 3.8 & 6.7 & 5.0 & 5.6 & 3.9 & 20.9 & 6.2 & 20.3 \\
\hline 11 & 3.7 & 6.2 & 5.4 & 6.9 & 1.9 & 20.2 & 4.1 & 16.3 \\
\hline 12 & 3.8 & 6.3 & 3.7 & 4.6 & 2.7 & 17.7 & 2.6 & 17.0 \\
\hline Mean(s.d.) & $3.8(0.1)$ & $6.4(0.2)$ & $5.3(1.2)$ & $5.9(0.9)$ & $2.6(0.8)$ & 19.3(1.3) & 4.8(1.5) & 19.3(2.9) \\
\hline \multicolumn{9}{|l|}{ Low/pla } \\
\hline 13 & 4.0 & 6.3 & 4.2 & 5.2 & 1.8 & 21.0 & 2.0 & 15.4 \\
\hline 14 & 5.5 & 7.5 & 3.4 & 7.6 & 0.9 & 21.9 & 0.6 & 21.3 \\
\hline 15 & 4.9 & 6.1 & 4.1 & 5.9 & 0.3 & 26.9 & 2.3 & 19.3 \\
\hline 16 & 5.0 & 7.7 & 4.6 & 7.1 & 0.2 & 16.8 & 0.5 & 19.2 \\
\hline Mean(s.d.) & $4.9(0.5)$ & $6.9(0.7)$ & $4.1(0.4)$ & $6.5(0.9)$ & $0.8(0.6)$ & $21.7(3.6)$ & $1.4(0.8)$ & $18.8(2.1)$ \\
\hline
\end{tabular}

Mod/glu, moderate carbohydrate diet and pre-exercise ingestion of glucose; Mod/pla, moderate carbohydrate diet and pre-exercise ingestion of a low-energy sweetened placebo; Low/glu, low dietary intake of carbohydrate and pre-exercise glucose ingestion; Low/pla, low carbohydrate diet and pre-exercise consumption of a sweetened low-energy placebo

pre-exercise glucose consumption influenced performance, and unlike our previous investigation ${ }^{4}$, the present low $\mathrm{CHO}$ (placebo) group recorded remarkably similar measures of total work during $\mathrm{MIT}_{1}$ and $\mathrm{MIT}_{2}$ (mean(s.d.) $111.7(13) \mathrm{kJ}$ and $113.1(12.5) \mathrm{kJ}$ respectively).

The 14 subjects in our previous study averaged a total of almost $140 \mathrm{~kJ}$ over their five work bouts considerably higher than the mean of $118 \mathrm{~kJ}$ achieved by the 16 subjects in the present investigation. Although $\dot{V}_{\mathrm{O}_{2} \text { ax }}$ differed by only $3.1 \mathrm{ml} \mathrm{kg}^{-1} \min ^{-1}$ between the two groups of volunteers, differences in the total work output in the interval test suggest a contrast in the subjects' ability for 'anaerobic' exercise. Although not entirely clear, this difference in ability may account for the disparity between the two investigations.

A number of previous studies have demonstrated the extent to which muscle glycogen can be significantly reduced in response to high-intensity interval exercise. For example, MacDougal et al. ${ }^{10}$ required their six subjects to cycle at $140 \% \dot{V}_{\mathrm{O}_{2} \text { max }}$ for as many repeated 60-s bouts as possible; each bout was separated by $3 \mathrm{~min}$ of recovery, and the exercise test was terminated when subjects were unable to maintain $30 \mathrm{~s}$ of continuous exercise. Time to fatigue ranged from 6 to $16 \mathrm{~min}$, and the authors reported that the more highly trained subjects achieved the greatest absolute power outputs but incurred the shortest total exercise times. Muscle glycogen sam- pled from the vastus lateralis at fatigue, was reduced by a mean of $70 \%$ from resting values. In a similar study which used less intense exercise, Thompson $e t$ al. ${ }^{11}$ required their subjects to cycle at $120 \% \mathrm{VO}_{2} \max$ for ten 60 -s bouts. Total muscle glycogen fell by an average of $52 \%$ from rest to completion of the tenth bout, while glycogen in the Type IIb fibres was reduced by $77 \%$. When one also considers that muscle glycogen can be reduced by as much as $25 \%$ following a single 30 -s sprint ${ }^{12}$, the exercise protocol and $\mathrm{CHO}$ restriction employed in the present study was expected to elicit significant changes in performance. Again with reference to differences in the ability for high intensity exercise, it is possible that when compared with those subjects involved in our previous study, the present 16 subjects differed with respect to their substrate utilization.

No differences in exercise $\dot{V}_{\mathrm{O}_{2}}$ were identified between $\mathrm{MIT}_{1}$ and $\mathrm{MIT}_{2}$ with respect to $\mathrm{CHO}$ intake. However, analysis of variance found that those subjects who had been administered the glucose solution before $\mathrm{MIT}_{2}$ consumed less total oxygen than those who were given the sweetened low-energy placebo $(P<0.05)$. An explanation for this finding is not readily apparent, especially as total work accomplished failed to change in response to the same treatment. Low subject numbers in each subgroup $(n=4)$, coupled with large standard deviations (Figure 4) also limit speculation. As plasma free fatty acids (FFAs) and glycerol were not measured, it 
is not possible to suggest that a shift in FFA utilization was related to the differences in $\dot{V} \mathrm{O}_{2}$. However, the subjects, even in their final exercise bout, averaged a power output close to $400 \mathrm{~W}$, at least equivalent to the intensity corresponding to their final $\dot{V}_{\mathrm{O}_{2} \max }$ work load. Although McCartney et al. ${ }^{13}$ have proposed that FFAs may contribute to the energy yield during such high intensity exercise, convention suggests that $\mathrm{CHO}$ and creatine phosphate are the only fuel sources capable of meeting such a high rate of adenosine triphosphate (ATP) resynthesis during exercise in which oxygen delivery to the muscles is compromised.

Peak plasma lactate and post-exercise blood $\mathrm{pH}$ were measured to detect possible differences in substrate utilization induced by the experimental procedures, but as neither variable significantly differed in response to dietary manipulation or solution administered, significant changes in substrate utilization during exercise remain questionable.

When the present data are considered in light of those from our previous investigation ${ }^{4}$, it appears possible that training status and/or 'sprint' ability may be significant factors determining the influence which dietary $\mathrm{CHO}$ exerts on supramaximal intermittent exercise performance. Further understanding of the physiological responses to exercise of this nature would benefit from before and after exercise measures of muscle glycogen to be coupled with both oxygen consumption and the estimated contribution of FFAs to the exercise demand.

\section{Acknowledgements}

The authors gratefully acknowledge the sponsorship of Kellogg Australia, the generosity of Mike Fatouris (Foss Electric Australia) and the statistical advice of Dr Alf Howard.

\section{References}

1 MacLaren DP, Gibson H, Parry-Billings M, Edwards RHT. A review of metabolic and physiological factors in fatigue. In: Pandolf KB, ed. Exercise and Sports Science Reviews. Baltimore, USA: Williams and Wilkins, Volume 17, 1989.

2 Maughan RJ, Poole DC. The effects of a glycogen loading regimen on the capacity to perform anaerobic exercise. Eur J Appl Physiol 1981; 46: 211-19.

3 Pizza FX, Flynn MG, Duscha B, Holden J, Kubitz ER. The effects of a glycogen supercompensation regimen on high intensity exercise performance. Med Sci Sports Exerc 1991; 23: S466.

4 Jenkins DG, Palmer JM, Spillman D. The influence of dietary carbohydrate on supramaximal intermittent exercise. Eur J Appl Physiol 1993; 67: 309-14.

5 Coyle EF, Coggan AR, Hemmert MK, Ivy JL. Muscle glycogen utilisation during prolonged strenuous exercise when fed carbohydrate. J Appl Physiol 1986; 61: 165-72.

6 Coggan AR, Coyle EF. Metabolism and performance following carbohydrate ingestion late in exercise. Med Sci Sports Exerc 1989; 21: 59-65.

7 Taylor HL, Buskirk E, Henschel A. Maximal oxygen uptake as an objective measure of cardio-respiratory performance. J Appl Physiol 1955; 8: 73-80.

8 McArdle WD, Katch FI, Katch VL. Exercise Physiology: Energy, Nutrition and Human Performance. Philadelphia, USA: Lea and Febiger, 1991.

9 Greenhaff PL, Gleeson M, Maughan RJ. The effects of dietary manipulation on blood acid-base status and the performance of high intensity exercise. Eur J Appl Physiol 1987; 56: 331-7.

10 MacDougal JD, Ward GR, Sale DG, Sutton JR. Muscle glycogen repletion after high-intensity intermittent exercise. J Appl Physiol 1977; 42: 129-132.

11 Thompson JA, Green HJ, Houston ME. Muscle glycogen depletion patterns in fast twitch fibre subgroups of man during submaximal and supramaximal exercise. Pflugers Arch 1979; 379: 105-8.

12 Boobis L, Williams C, Wootton SA. Human muscle metabolism during brief maximal exercise. J Physiol (Lond) 1982; 338: 21-22 (Abstract).

13 McCartney N, Spriet LL, Heigenhauser GJ, Kowalchuk JM, Sutton JR, Jones NL. Human power and metabolism in maximal intermittent exercise. J Appl Physiol 1986; 60: 1164-9. 